\title{
Evaluation of spine structure stability at different locations during SBRT
}

\author{
Lukas Knybel ${ }^{\mathrm{a}}$, Jakub Cvek ${ }^{\mathrm{a}}$, Zuzana Cermakovaa , Jaroslav Havelka ${ }^{\mathrm{b}}$, Michaela Pomaki ${ }^{\mathrm{b}}$, Kamila Resova ${ }^{\mathrm{a}}$
}

\begin{abstract}
Background and Aims. Modern stereotactic body radiotherapy (SBRT) techniques and systems that use online image guidance offer frameless radiotherapy of spinal tumors and the ability to control intrafraction motion during treatment. These systems allow precise alignment of the patient during the entire treatment session and react immediately to random changes in this alignment. Online tracking data provide information about intrafractional changes, and this information can be useful for designing treatment strategies even if online tracking is not being used. The present study evaluated spine motion during SBRT treatment to assess the risk of verifying patient alignment only prior to starting treatment.
\end{abstract}

Methods. This study included 123 patients treated with spine SBRT. We analyzed different locations within the spine using system log files generated during treatment, which contain information about differences in the pretreatment reference spine positions by CT versus positions during SBRT treatment. The mean spine motion and intra/interfraction motion was evaluated. We defined and assessed the spine stability and spine significant shifts ( $\mathrm{SSH}$ s) during treatment. Results. We analyzed 462 fractions. For the cervical (C) spine, the greatest shifts were in the anterior-posterior (AP) direction $(2.48 \mathrm{~mm})$ and in pitch rotation $(1.75 \mathrm{deg})$. The thoracic $(\mathrm{Th})$ spine showed the biggest shift in the AP direction $(3.68 \mathrm{~mm})$ and in roll rotation (1.66 deg). For the lumbar-sacral (LS) spine, the biggest shift was found for left-right (LR) translation $(3.81 \mathrm{~mm}$ ) and roll rotation $(3.67 \mathrm{deg})$. No C spine case exceeded $1 \mathrm{~mm} / 1$ deg for interfraction variability, but 7 of 54 Th spine cases exceeded $1 \mathrm{~mm}$ interfraction variability for translations (maximum value, $2.5 \mathrm{~mm}$ in the AP direction). The interfraction variability for translations exceeded $1 \mathrm{~mm}$ in 2 of $24 \mathrm{LS}$ spine cases (maximum value, $1.7 \mathrm{~mm}$ in the LR direction). Only $13 \%$ of cases had no SSHs. The mean times to SSH were $6.5 \pm 3.9 \mathrm{~min}, 8.1 \pm 5.9 \mathrm{~min}$, and $8.8 \pm 7.1 \mathrm{~min}$ for the $\mathrm{C}$, Th, and LS spine, respectively, and the mean recorded SSH values were 1.6 $\pm 0.66,1.43 \pm 0.33$, and $1.46 \pm 0.47 \mathrm{~mm} / \mathrm{deg}$, respectively.

Conclusion. Positional tracking during spine SBRT treatments revealed low mean translational and rotational shifts. Patient immobilization did not improve spine shifts compared with our results for the Th and LS spine without immobilization. For the most precise spine SBRT, we recommend checking the patient's position during treatment.

Key words: spine, SBRT, intrafraction variability

Received: March 21, 2019; Accepted: May 30, 2019; Available online: June, 17, 2019

https://doi.org/10.5507/bp.2019.027

(c) 2020 The Authors; https://creativecommons.org/licenses/by/4.0/

${ }^{a}$ Department of Oncology, University Hospital Ostrava, Ostrava, Czech Republic

${ }^{b}$ Department of Radiology, University Hospital Ostrava, Ostrava, Czech Republic

Corresponding author: Jakub Cvek, e-mail:jakub.cvek@fno.cz

\section{BACKGROUND}

Stereotactic body radiotherapy (SBRT) requires high accuracy in order to deliver high doses of radiation to areas close to the organs at risk, i.e. the spinal cord and cauda equina. Higher single doses produce better clinical outcomes ${ }^{1-3}$, but both single and multifraction regimens are safe and efficacious in spine SBRT for spinal metasta$\mathrm{ses}^{4}$. SBRT of spine tumors is challenging because of the close relationship between the target tissue and the spinal cord, and possible errors during treatment, especially patient/target motion, must be minimized. Radiation myelopathy manifests clinically as slowly progressing severe sensorimotor impairment that can include paraplegia and quadriplegia. The latency period for the onset of myelopathy is typically 6 months, but it can occur up to 20 months after radiation therapy. Although this toxicity is rare, the consequences to the patient are devastating ${ }^{5}$.
SBRT treatments can have longer treatment times than conventional treatments and use steep dose gradients. Therefore, the dose that is delivered to the spinal cord can be greatly affected by even small movements of the patient and thus of related spinal structures, as shown in previous studies ${ }^{6-9}$.

Modern SBRT techniques and systems that use online image guidance offer frameless radiotherapy of spinal tumors and the ability to control intrafraction motion during treatment. These systems allow precise alignment of the patient during the entire treatment session and react immediately to random changes in this alignment ${ }^{10}$. Online tracking data provide information about intrafractional changes, and this information can be useful for designing treatment strategies even if online tracking is not being used. The present study evaluated spine motion during SBRT treatment to assess the risk of verifying patient alignment only prior to starting treatment. 


\section{METHODS}

This study included 123 patients who were treated with spine SBRT in 1, 3, or 5 fractions. The data were from all regions of the spine: 36 cases of SBRT of the cervical region ( $\mathrm{C}$ spine), 58 cases of SBRT of the thoracic region (Th spine), and 29 cases of SBRT of the lumbar-sacral region (LS spine). We used the CyberKnife ${ }^{\circledR}$ system and Xsight $^{\mathrm{TM}}$ spine tracking technology. This methodology has been described in detail previously ${ }^{11-13}$ and has sub-millimeter translational accuracy ${ }^{10,14}$. In brief, the CyberKnife ${ }^{\circledR}$ system uses two X-ray sources to obtain orthogonal live images of spine structures, which are then compared with a pair of digitally reconstructed radiographs (DRRs) that are generated from the planning CT. Three to four adjacent vertebrae are usually included in the grid, which is matched in the reference DRRs and live images. The grid nodes are then compared, and the displacement of the target is computed from two 2D displacement fields ${ }^{12}$. Interpolation allows the calculation of $3 \mathrm{D}$ displacement and rotation.

Live images were taken every $60 \mathrm{~s}$ after successful patient alignment relative to the reference DRRs. All treatments began only after the differences between the reference spinal positions and the actual spine positions in the region of interest differed by less than $1 \mathrm{~mm}$ and by less than $1 \mathrm{deg}$. Notably, information about the difference between the spine position on DRRs and on live images is saved in the system log files. Translations in the superior-inferior (SI), left-right (LR), and anteriorposterior (AP) directions and rotations are called yaw (clockwise/counterclockwise), pitch (head up/down), and roll, respectively, and were evaluated using the log files. The treatment system distinguishes the directions of movement based on positive and negative signs. Thus, positive $(+)$ values indicate that the spine position is more superior, right, posterior, clockwise, head down, or rolled left than on the reference DRR image. The following parameters were defined to describe spine motion during SBRT treatment.

\section{Spine stability}

To evaluate intrafractional motion, we defined "spine stability" as the mean absolute difference between translations/rotations that were recorded at the start of treatment versus translations/rotations recorded after each image acquisition. If treatment was interrupted for some reason (for example, if the patient moved), a new starting position was recorded and used for subsequent comparisons.

\section{Interfraction motion}

Interfraction variability was defined as the standard deviation (SD) of the mean spine translation or rotation over the entire treatment session.

\section{Spine significant shift (SSH)}

For this parameter, we recorded the time from the start of the treatment until the time at which the tracking area suddenly shifted more than $1 \mathrm{~mm}$ or $1 \mathrm{deg}$ in any direction. We considered $1 \mathrm{~mm}$ or $1 \mathrm{deg}$ to be an $\mathrm{SSH}$ relative to the planned target coverage, in agreement with Chuang et al. ${ }^{6}$.

\section{RESULTS}

A total of 462 fractions were analyzed, and the mean treatment time was $30 \mathrm{~min}$ (range 15-47 $\mathrm{min}$ ). Table 1 shows the translations and rotations of the $\mathrm{C}$, Th, and LS regions of the spine. The mean values were very low because of compensatory spine movements i.e. superior and inferior movements, left and right movements, etc.

The $\mathrm{C}$ spine showed the highest shifts in pitch rotation (range -0.91-1.04 deg; head up to head down). The Th spine structures showed the highest AP translations during treatment (range -2.1-5.6; anterior to posterior). The LS spine showed the highest inferior translations ( $\max -3.4 \mathrm{~mm}$ ) and LR translations (range -2.1-2.8; left to right)

\section{Interfraction variability}

For the 111 patients whose treatment involved more than 1 fraction, the interfraction spine motion was evaluated as the SD of the mean spine translation or rotation between fractions. The interfraction variability was less than $1 \mathrm{~mm}$ or $1 \mathrm{deg}$ for all of the $\mathrm{C}$ spine cases. The interfraction variability for translations was greater than $1 \mathrm{~mm}$ in 7 out of $54 \mathrm{Th}$ spine cases, and it showed a maximum value of $2.5 \mathrm{~mm}$ in the AP direction. The interfraction variability for translations was greater than $1 \mathrm{~mm}$ in 2 out of $24 \mathrm{LS}$ spine cases, with a maximum value of $1.7 \mathrm{~mm}$ in the LR direction.

\section{Spine stability}

Spine stability describes the rate of translation and rotation during treatment relative to the first alignment of the patient or relative to the next alignment if treatment was interrupted and the patient was realigned. All of the

Table 1. The translation and rotation of the cervical (C), thoracic (Th) and lumbar-sacral (LS) regions of the spine during SBRT treatment.

\begin{tabular}{|c|c|c|c|c|c|c|}
\hline & \multicolumn{3}{|c|}{ Translation, $\mathrm{mm}$} & \multicolumn{3}{|c|}{ Rotation, degree } \\
\hline & $\mathrm{SI}(\mathrm{S}+)$ & $\mathrm{LR}(\mathrm{R}+)$ & $\mathrm{AP}(\mathrm{P}+)$ & Yaw $(\mathrm{CW}+)$ & Pitch (head down + ) & Roll (rolled left + ) \\
\hline C spine & $-0.12 \pm 0.43$ & $0.06 \pm 0.72$ & $-0.13 \pm 0.76$ & $0.02 \pm 0.44$ & $-0.12 \pm 0.42$ & $0.08 \pm 0.58$ \\
\hline Th spine & $-0.16 \pm 0.49$ & $-0.17 \pm 0.74$ & $0.23 \pm 0.46$ & $0.10 \pm 0.33$ & $0.04 \pm 0.25$ & $-0.01 \pm 0.39$ \\
\hline LS spine & $-0.70 \pm 0.55$ & $0.25 \pm 0.71$ & $0.07 \pm 0.69$ & $0.02 \pm 0.23$ & $0.14 \pm 0.25$ & $-0.05 \pm 0.33$ \\
\hline
\end{tabular}

Values are reported as means \pm standard deviations.

$\mathrm{SI}=$ superior-inferior; $\mathrm{LR}=$ left-right; $\mathrm{AP}=$ anterior-posterior, $\mathrm{CW}=$ clockwise 

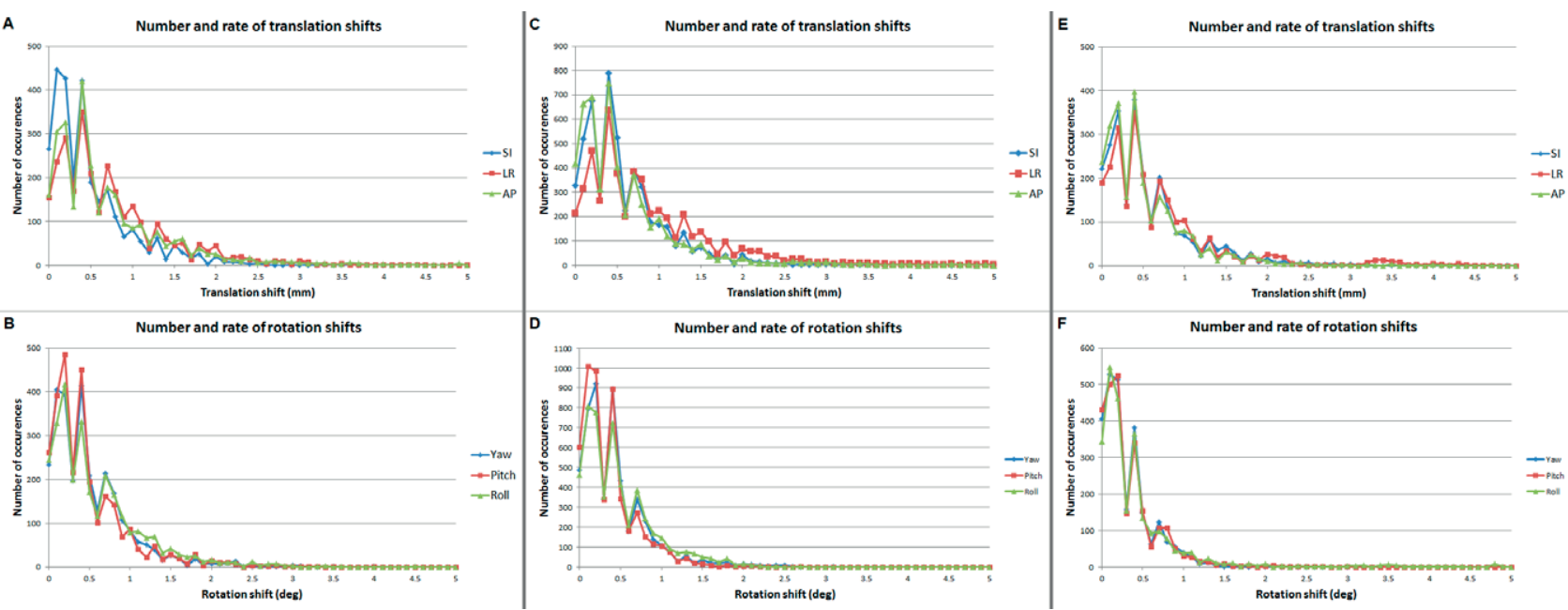

Fig. 1. The number and rate of spine shifts for the cervical $(A, B)$, thoracic $(C, D)$ and lumbar-sacral regions of the spine (E, F).

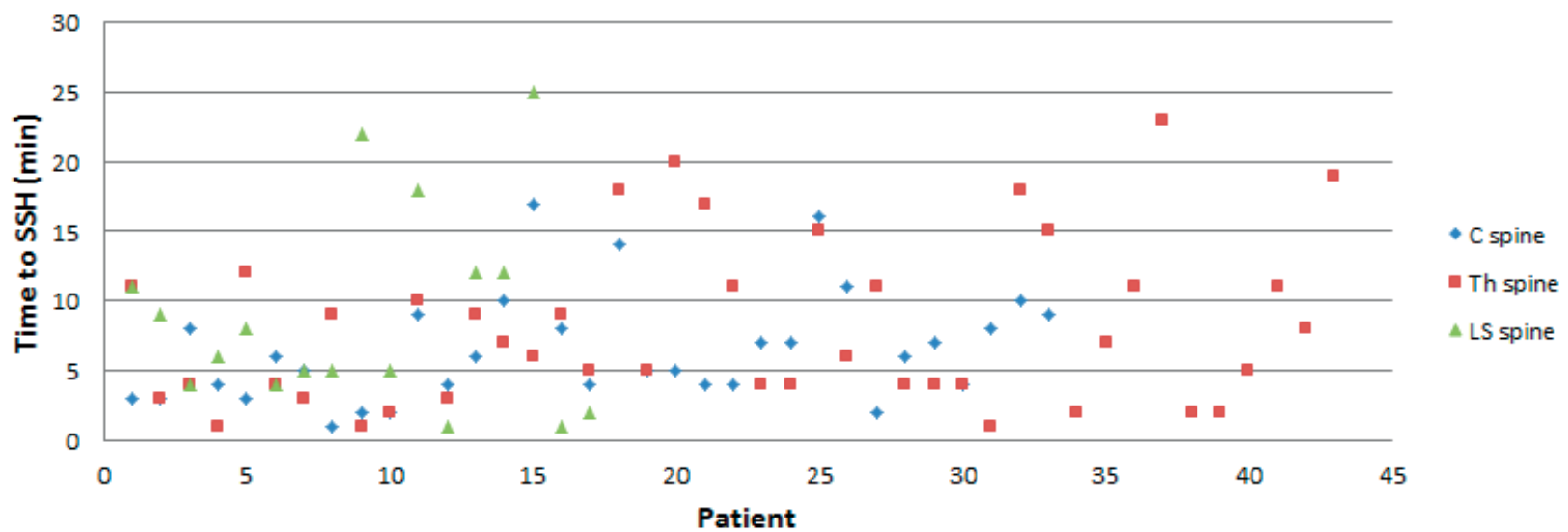

Fig. 2. Time to spine significant shift (SSH) for the cervical (C spine), thoracic (Th spine), and lumbar-sacral (LS spine) regions of the spine. Some points overlapped.

mean translation and rotation values were less than $1 \mathrm{~mm}$ and $1 \mathrm{deg}$, except for LR translations in the Th spine. For the $\mathrm{C}$ spine, we observed the maximum shifts in the AP direction $(2.48 \mathrm{~mm})$ and in pitch rotation $(1.75 \mathrm{deg})$. The Th spine showed the biggest shift in the AP direction $(3.68 \mathrm{~mm})$ and in roll rotation $(1.66 \mathrm{deg})$. For the LS spine, we detected the biggest shift in LR translation $(3.81 \mathrm{~mm})$ and in roll rotation (3.67 deg). Fig. 1 show the number of relevant shifts for the $\mathrm{C}$, Th, and LS spine, respectively, in 3 translation and 3 rotation directions.

\section{SSH}

We set the threshold for SSH at $1 \mathrm{~mm}$ (translation) or 1 degree (rotation) from the start of treatment and evaluated the time at which the threshold was exceeded. Spine position was recorded according to imaging frequency, which was every $60 \mathrm{~s}$. We only evaluated the first fraction for each patient. Just 16 (13\%) of the 123 spine cases did not exceed SSH during delivery of the entire fraction (range 13-39 $\mathrm{min}$ ). The mean time to $\mathrm{SSH}$ was $6.5 \pm 3.9$ min (range 1-17 $\mathrm{min}$ ), 8.1 $\pm 5.9 \mathrm{~min}$ (range 1-23 $\mathrm{min}$ ), and $8.8 \pm 7.1 \mathrm{~min}$ (range 1-25 $\mathrm{min}$ ) for the $\mathrm{C}$, Th, and LS spine, respectively.

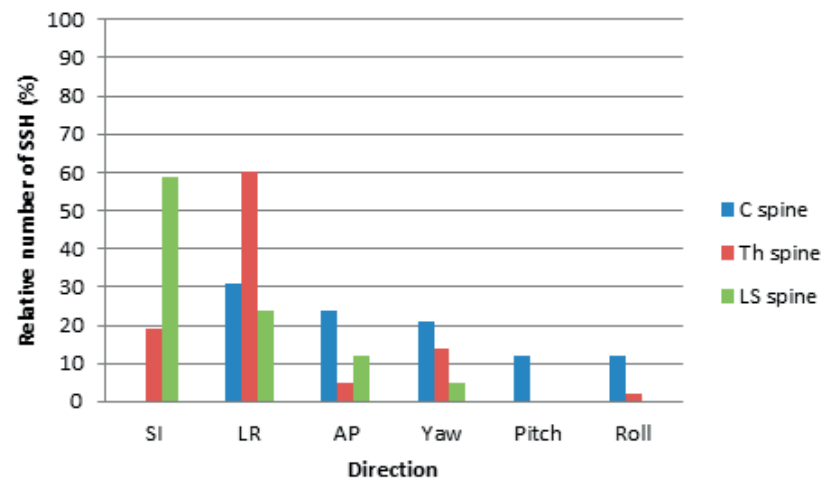

Fig. 3. The relative occurrence (\%) of spine significant shifts (SSHs) in translational and rotational directions for the cervical (C), thoracic (Th), and lumbar-sacral (LS) regions of the spine.

The mean recorded SSH was $1.6 \pm 0.66,1.43 \pm 0.33$ and $1.46 \pm 0.47 \mathrm{~mm} / \mathrm{deg}$ for the $\mathrm{C}$, Th, and LS spine respectively. Figure 3 shows the frequency of shifts that exceeded the $1 \mathrm{~mm} / \mathrm{deg}$ threshold. The number of shifts was recalculated as a percentage to compare $\mathrm{C}$, Th, and LS groups using the same scale. The Th spine had the 
most SSHs in the LR direction, and the LS spine had the most SSHs in the SI direction.

\section{DISCUSSION}

Stereotactic irradiation is a safe and effective treatment option for tumors of the spine and spinal cord ${ }^{15}$. Treatment accuracy is very important in order to deliver high doses of radiation only to the target region, which is near critical structures, such as the spinal cord. The CyberKnife ${ }^{\circledR}$ system allows periodic monitoring of the spine during treatment. Patient movement is monitored, recorded, and compensated for by adjusting the beam direction via the robotic arm holding the linear accelerator. Treatment data allow the spine motion to be evaluated in the area that is being treated throughout the entire treatment, and these data can be a good source of information when periodic monitoring is not possible.

The primary aims of this study were to evaluate spine motion in different spine locations in a large sample of cases during SBRT and to evaluate the time at which SSH occurred. Even a small movemet during SBRT can significantly affect the dose that is delivered to the spinal cord $^{6-9}$. We evaluated both translational and rotational movement, since cases in which the target is close to the spinal cord can benefit from corrections in rotation ${ }^{16}$. Here we used thermoplastic head fixation for all $\mathrm{C}$ spine cases; all other treatments were performed without special fixation with the patient lying on a soft pad that was comfortable but that did not fix the patient 's body in a particular position.

The mean spine motion value during treatment was very low ( $<1 \mathrm{~mm}$ or $1 \mathrm{deg}$ in all cases), which is in agreement with previous studies ${ }^{17-20}$. This mean value did not reflect "real" motion because of compensatory spine movements i.e. superior and inferior, left and right, etc.; that is, averaging the results gave a net result that was close to zero. To better describe possible spine motion during SBRT, we defined spine stability as the mean absolute difference between translations/rotations recorded at the start of the treatment versus translations/rotations recorded after each image acquisition. Only the mean value for the Th spine in the LR direction exceeded $1 \mathrm{~mm}$. Hyde et al. ${ }^{17}$ also reported that the highest number of positioning errors were in the LR direction, although their study did not distinguish between spine regions. Our mean spine motion values were low, although larger individual shifts were detected, with maximum values in the LS spine (3.81 $\mathrm{mm}$ for LR translation and $3.67 \mathrm{deg}$ for roll rotation). Interestingly, Jin et al. ${ }^{21}$ reported lower mean values for intrafraction translational motion and rotational motion but higher SD values. This may indicate that their patients showed higher random shifts in positions compared to the patients in our study. All of our patients were not immobilized during treatment, and our results are in agreement with those of Hoogeman et al. ${ }^{22}$, who found that patient immobilization does not guarantee that patient movement is less than several millimeters.

The overall interfraction variability was low in our study, with just $13 \%$ of Th spine cases and $8 \%$ of LS spine cases exceeding $1 \mathrm{~mm}$ interfraction variability for translational motion. $\mathrm{C}$ spine cases had the lowest translation interfraction variability, which may be because thermoplastic head fixation was used during treatment for $\mathrm{C}$ spine cases. Obviously the difference is not clinicaly significant (Table 2). We also evaluated SSH based on a $1 \mathrm{~mm} / 1 \mathrm{deg}$ threshold and found that $87 \%$ of the cases exceeded the SSH threshold during the treatment. Murphy et al. ${ }^{20}$ reported that $58 \%$ of their cases had at least one translational shift of more than $2 \mathrm{~mm}$. The maximum time without detection of SSH was 39 min in our study; Hyde et al. reported that patients remained within a tolerance range of $1 \mathrm{~mm} / 1 \mathrm{deg}$ for an average of $33 \pm 15 \mathrm{~min}\left(\right.$ ref. $\left.^{17}\right)$.

Table 2. Interfraction spine variability for translations and rotations during SBRT treatment in the cervical (C), thoracic (Th) and lumbar-sacral (LS) regions of the spine.

\begin{tabular}{lccc:ccc}
\hline & \multicolumn{3}{c}{ Translation, mm } & \multicolumn{3}{c}{ Rotation, deg } \\
& SI & LR & AP & Yaw & Pitch & Roll \\
\hline C spine & $0.29 \pm 0.20$ & $0.40 \pm 0.26$ & $0.31 \pm 0.22$ & $0.28 \pm 0.14$ & $0.34 \pm 0.20$ & $0.21 \pm 0.10$ \\
Th spine & $0.36 \pm 0.26$ & $0.56 \pm 0.41$ & $0.38 \pm 0.37$ & $0.25 \pm 0.15$ & $0.25 \pm 0.18$ & $0.29 \pm 0.16$ \\
LS spine & $0.52 \pm 0.34$ & $0.59 \pm 0.38$ & $0.44 \pm 0.30$ & $0.18 \pm 0.09$ & $0.23 \pm 0.13$ & $0.27 \pm 0.2$ \\
\hline
\end{tabular}

Values are reported as means \pm standard deviations.

$\mathrm{SI}=$ superior-inferior; $\mathrm{LR}=$ left-right; $\mathrm{AP}$ = anterior-posterior.

Table 3. Spine stability during SBRT treatment in the cervical (C), thoracic (Th) and lumbar-sacral (LS) regions of the spine.

\begin{tabular}{|c|c|c|c|c|c|c|}
\hline & \multicolumn{3}{|c|}{ Translation, $\mathrm{mm}$} & \multicolumn{3}{|c|}{ Rotation, deg } \\
\hline & $\mathrm{SI}, \mathrm{mm}$ & LR & AP & Yaw & Pitch & Roll \\
\hline C spine & $0.48 \pm 0.32$ & $0.74 \pm 0.50$ & $0.72 \pm 0.50$ & $0.48 \pm 0.32$ & $0.45 \pm 0.29$ & $0.56 \pm 0.43$ \\
\hline Th spine & $0.67 \pm 0.48$ & $1.05 \pm 0.71$ & $0.72 \pm 0.58$ & $0.42 \pm 0.26$ & $0.35 \pm 0.21$ & $0.47 \pm 0.30$ \\
\hline LS spine & $0.66 \pm 0.52$ & $0.81 \pm 0.72$ & $0.67 \pm 0.66$ & $0.29 \pm 0.18$ & $0.31 \pm 0.20$ & $0.42 \pm 0.26$ \\
\hline
\end{tabular}

Values are reported as means \pm standard deviations.

$\mathrm{SI}=$ superior-inferior; $\mathrm{LR}=$ left-right; $\mathrm{AP}=$ anterior-posterior. 
The mean time to SSH was longer than $6 \mathrm{~min}$ in all spine segments, giving a probable motion of less than 0.17 $\mathrm{mm} / \mathrm{min}$. Kim et al. ${ }^{23}$ evaluated the intra-treatment spinal motion pre-, mid- and post-treatment for cone beam CT $(\mathrm{CBCT})$ and reported the potential magnitude of motion per unit time as $0.15 \mathrm{~mm} / \mathrm{min}$, which is in agreement with our results.

Fig. 3 shows the relative number of SSHs for the $\mathrm{C}$, Th, and LS spine. The $\mathrm{C}$ spine had a similar number of SSHs for all translations and rotations, except for the SI direction, in which no SSH was detected. Ma et al. ${ }^{13}$ showed that the $\mathrm{C}$ spine is unique in that is has intrafractional variations along all six degrees of freedom. In contrast, the Th spine and the LS spine had the majority of SSHs in the LR direction and the SI direction, respectively.

In this study, we evaluated the motion of different spine structures during SBRT. To best of our knowledge, this is the largest patient sample to be evaluated this way using online tracking. We evaluated the most common parameters describing spine shifts. Moreover SSH was based on a strict $1 \mathrm{~mm} / 1$ deg threshold which is more reasonable for precise SBRT than higher threshold published in past. Our patients had no fixation system except for C spine cases, which used a thermoplastic mask. The results demonstrate that spine position changes with time, and we agree with Hoogeman et al..$^{22}$ that the patient slowly drifts away from the initial position during treatment.

\section{CONCLUSION}

In conclusion, online tracking of spine movement during spine SBRT treatments revealed low mean translational and rotational shifts. Patient immobilization did not improve spine shifts in the $\mathrm{C}$ spine compared to results in the Th and LS spine, for which fixation was not used. To obtain the most precise results with spine SBRT, we recommend checking the patient's position during treatment and keeping the treatment time short to prevent significant shifts in the spine. We detected the largest number of significant shifts in the LR and SI directions for the Th and LS spine.

\section{ABBREVIATIONS}

AP, Anterior - posterior; C, Cervical (spine); CBCT, Cone beam CT; DRR, Digitally reconstructed radiograph; LR, Left- right; LS, Lumbar-sacral (spine); SBRT, Stereotactic body radiotherapy; SD, Standard deviation; SI, Superior- inferior; SSH, Spine significant shift; Th, Thoracic (spine).

Acknowledgement: This work was supported by Intitutional support - RVO-FNOs/2016.

Author contributions: LK: data analysis, manuscript writing; JC: study design, manuscript writing; ZC: participated in data analysis and wrote specific part of manuscript; MP, JH: data collection; JH: participated in study design and coordination; KR: carried out a critical review of the manuscript; All authors read and approved the final manuscript.

Conflicts of interest statement: The authors state that there are no conflicts of interest regarding the publication of this article.

\section{REFERENCES}

1. Gerszten PC, Burton, SA, Ozhasoglu C, Vogel WJ, Welch WC, Baar J, Friedland DF. Stereotactic radiosurgery for spinal metastases from renal cell carcinoma. J Neurosurg Spine 2005;3(4):288-95.

2. Gerszten PC, Burton SA, Belani CP, Ramalingam S, Friedland DM, Ozhasoglu C, Welch WC. Radiosurgery for the treatment of spinal lung metastases. Cancer 2006;107(11):2653-61.

3. Jin JY, Chen Q, Jin R, Rock J, Anderson J, Li S, Ryu S. Technical and clinical experience with spine radiosurgery: a new technology for management of localized spine metastases. Technol Cancer Res Treat 2007;6(2):127-33.

4. Huo M, Sahgal A, Pryor D, Redmond K, Lo S, Foote M. Stereotactic spine radiosurgery: Review of safety and efficacy with respect to dose and fractionation. Surg Neurol Int 2017;8:30.

5. Abbatucci JS, Delozier T, Quint R, Roussel A, Brune D.Radiation myelopathy of the cervical spinal cord: time, dose and volume factors. Int J Radiat Oncol Biol Phys 1978;4(3):239-48.

6. Chuang C, Sahgal A, Lee L, Larson D, Huang K, Petti P, Ma L. Effects of residual target motion for image-tracked spine radiosurgery. Med Phys 2007;34(11):4484-90.

7. Guckenberger M, Meyer J, Wilbert J, Baier K, Bratengeier K, Vordermar, D, Flentje M. Precision required for dose-escalated treatment of spinal metastases and implications for image-guided radiation therapy (IGRT). Radiother Oncol 2007;84(1):56-63.

8. Wang H, Shiu A, Wang C, O'Daniel J, Mahajan A, Woo S, Chang EL. Dosimetric effect of translational and rotational errors for patients undergoing image-guided stereotactic body radiotherapy for spinal metastases. Int J Radiat Oncol Biol Phys 2008;71(4):1261-71.

9. Chawla S, Schell MC, Milano MT. Stereotactic body radiation for the spine: a review. Am J Clin Oncol 2013;36(6):630-36.

10. Fürweger $C$, Drexler $C$, Kufeld M, Muacevic A, Wowra B. Advances in fiducial-free image-guidance for spinal radiosurgery with CyberKnife-a phantom study. J Appl Clin Med Phys 2011;12(2):2028.

11. Fu D, Kuduvalli G, Maurer CR, Allision JW, Adler JR. 3D target localization using 2D local displacements of skeletal structures in orthogonal X-ray images for image-guided spinal radiosurgery. Int J Comput Assist Radiol Surg 2006;1:198-200.

12. Muacevic A, Staehler M, Drexler C, Wowra B, Reiser M, Tonn JC. Technical description, phantom accuracy, and clinical feasibility for fiducial-free frameless real-time image-guided spinal radiosurgery. J Neurosurg Spine 2006;5(4):303-12.

13. Ma L, Sahgal A, Hossain S, Chuang C, Descovich M, Huang K, Larson DA. Nonrandom intrafraction target motions and general strategy for correction of spine stereotactic body radiotherapy. Int J Radiat Oncol Biol Phys 2009;75(4):1261-65.

14. Ho AK, Fu D, Cotrutz C, Hancock SL, Chang SD, Gibbs IC, Adler JR. A study of the accuracy of cyberknife spinal radiosurgery using skeletal structure tracking. Neurosurgery 2007;60(suppl_2):ONS-147.

15. Elibe E, Boyce-Fappiano D, Ryu S, Siddiqui MS, Lee I, Rock J, Siddiqui F. Stereotactic radiosurgery for primary tumors of the spine and spinal cord. J Radiosurg SBRT 2018;5(2):107.

16. Li W, Sahgal A, Foote M, Millar BA, Jaffray DA, Letourneau D. Impact of immobilization on intrafraction motion for spine stereotactic body radiotherapy using cone beam computed tomography. Int J Radiat Oncol Biol Phys 2012;84(2):520-6.

17. Hyde D, Lochray F, Korol R, Davidson M, Wong CS, Ma L, Sahgal A. Spine stereotactic body radiotherapy utilizing cone-beam CT image-guidance with a robotic couch: intrafraction motion analysis accounting for all six degrees of freedom. Int J Radiat Oncol Biol Phys 2012;82(3):e555-62.

18. Descovich M, Ma L, Chuang CF, Larson DA, Barani IJ. Comparison 
between prone and supine patient setup for spine stereotactic body radiosurgery. Technol Cancer Res Treat 2012;11(3):229-36.

19. Agazaryan N, Tenn SE, Desalles AA, Selch MT. Image-guided radiosurgery for spinal tumors: methods, accuracy and patient intrafraction motion. Phys Med Biol 2008;53(6):1715.

20. Murphy MJ, Chang SD, Gibbs IC, Le QT, Hai J, Kim D, Adler Jr JR. Patterns of patient movement during frameless image-guided radiosurgery. Int J Radiat Oncol Biol Phys 2003;55(5):1400-08.
21. Jin JY, Ryu S, Rock J, Faber K, Chen Q, Ajlouni M, Movsas B. Evaluation of residual patient position variation for spinal radiosurgery using the Novalis image guided system. Mel Phys 2008,35(3):1087-93.

22. Hoogeman MS, Nuyttens JJ, Levendag PC, Heijmen BJ. Time dependence of intrafraction patient motion assessed by repeat stereoscopic imaging. Int J Radiat Oncol Biol Phys 2008;70(2):609-18.

23. Kim J, Hsia AT, Xu Z, Ryu S. Motion Likelihood Over Spine Radiosurgery Treatments-An Intrafraction Motion Analysis. Int J Radiat Oncol Biol Phys 2017,99(2):E678. 